\title{
O.S.P.
}

L'orientation scolaire et professionnelle

$33 / 4 \mid 2004$

Travail biographique, construction de soi et formation

$-1$

\section{Biographie, socialisation, formation}

Comment les individus deviennent-ils des individus?

Biography, socialization and development: How individuals grow up as

individuals?

\section{Christine Delory-Momberger}

\section{OpenEdition}

12 Journals

Édition électronique

URL : http://journals.openedition.org/osp/251

DOI : 10.4000/osp.251

ISSN : 2104-3795

Éditeur

Institut national d'étude du travail et d'orientation professionnelle (INETOP)

Édition imprimée

Date de publication : 15 décembre 2004

Pagination : $551-570$

ISSN : 0249-6739

Référence électronique

Christine Delory-Momberger, « Biographie, socialisation, formation », L'orientation scolaire et professionnelle [En ligne], 33/4 | 2004, mis en ligne le 28 septembre 2009, consulté le 16 décembre 2020. URL : http://journals.openedition.org/osp/251 ; DOI : https://doi.org/10.4000/osp.251

Ce document a été généré automatiquement le 16 décembre 2020.

(c) Tous droits réservés 


\title{
Biographie, socialisation, formation
}

\author{
Comment les individus deviennent-ils des individus? \\ Biography, socialization and development: How individuals grow up as \\ individuals?
}

Christine Delory-Momberger

1 En tant qu'ensemble construit des représentations que l'individu se fait de sa propre vie et de son histoire, la biographie est ainsi devenue une composante et un horizon du champ de la formation. Comment les individus, jeunes et adultes, intègrent-ils, dans leurs représentations d'eux-mêmes et de leur existence, les expériences d'apprentissage et de formation qu'ils connaissent aux différents âges de leur vie ? À quelles formes de travail biographique recourent-ils pour s'approprier les épisodes de formation que les formes contemporaines de la vie sociale contribuent à déployer tout au long de la vie? Pour saisir la relation constitutive entre biographie et formation et échapper aux allant-de-soi auxquels elle prête trop facilement, on ne peut faire l'économie d'une réflexion sur ce qui constitue l'espace du biographique, sur les formes d'activité et d'opérativité auxquelles il correspond, sur le rôle qu'il joue dans les processus d'individuation et de socialisation.

\section{Biographie et socialisation}

2 S'interroger sur le biographique, c'est s'interroger sur la manière dont les individus accèdent à leur vécu, dont ils se saisissent des situations, des événements, des rencontres, des émotions que la vie leur apporte. L'expérience la plus immédiate montre que nous n'avons pas un accès direct à notre vécu, que nous n'y atteignons qu'à travers la médiation des mots et des représentations que nous posons sur lui, et que ces mots et ces représentations prennent forme dans des « histoires » (Schapp, 1992). Dès que nous voulons nous saisir de notre vie, nous la racontons; nous n'avons pas d'autre moyen pour accéder à notre vie que de percevoir ce que nous vivons à travers l'écriture d'une histoire ou d'une multiplicité d'histoires : en quelque sorte nous ne vivons notre 
vie qu'autant que nous la biographions, que nous lui donnons, au sens étymologique du terme, la forme d'une écriture (Delory-Momberger, 2003).

3 Le récit, sous l'espèce de l'« histoire de vie » ou sous la forme des fragments narratifs qui constituent très souvent le fond de nos échanges quotidiens avec autrui, apparaît comme la forme la plus familière de cette écriture du vécu. Par le récit, nous transformons les événements, les actions et les personnes de notre vie en épisodes, en intrigues et en personnages; nous construisons entre eux des relations finalisées entre un commencement et une fin; nous leur assignons une place dans des ensembles construits et orientés selon une logique narrative (Ricœur, 1983, p. 102). Par le récit, nous nous faisons le propre personnage de notre vie et nous donnons à celle-ci une histoire. Autrement dit, nous ne faisons pas le récit de notre vie parce que nous avons une histoire; mais au contraire, nous avons une histoire parce que nous faisons le récit de notre vie.

\section{Le biographique, une catégorie de l'expérience}

Cependant le discours biographique, écrit ou parlé, suivi ou parcellaire, n'est que le territoire émergent et spectaculaire d'un continent plus vaste, qui s'enracine dans une attitude plus fondamentale de l'être humain : celle qui consiste à se représenter son existence dans le temps et à configurer narrativement la succession temporelle de son expérience. L'« écriture de la vie ", à laquelle renvoient l'étymologie et le sens commun du mot biographie, doit être ici entendue comme une attitude première et spécifique du vécu humain : avant même de laisser de notre vie une quelconque trace écrite, avant tout discours, oral ou écrit, tenu sur nous-même, nous écrivons mentalement notre vie. La perception et l'intelligence de notre vécu passent par des représentations qui prêtent une figure aux événements et aux situations de notre existence. Les images dont nous nous servons pour évoquer la vie (ligne, fil, trajet, chemin, parcours, cercle, cycle, carrière de la vie) nous sont cependant tellement familières qu'elles ont perdu leur dimension symbolique ou analogique et qu'elles en viennent à masquer le fait biographique lui-même, autrement dit l'acte même de la représentation, et à faire oublier que ce n'est que par métaphore, selon les voies d'une écriture, que de telles figures peuvent être prêtées à notre existence.

4 Cette capacité anthropologique selon laquelle les hommes perçoivent leur vie et ordonnent leur expérience dans les termes d'une raison narrative constitue le fait biographique premier. Sur lui se construisent tous les espaces des histoires humaines: celui de la rétrospection de la vie passée, celui de l'anticipation de l'heure ou du jour à venir, celui de la projection de la vie future, ceux des aventures les plus rares et singulières comme des faits les plus quotidiens et routiniers. Nous ne cessons de fait de nous biographier, c'est-à-dire d'inscrire notre expérience dans des schémas temporels orientés qui organisent mentalement nos gestes, nos comportements, nos actions, selon une logique de configuration narrative. Dans le quotidien de notre vie, un grand nombre de ces opérations de biographisation sont douées d'une relative automaticité et peuvent ne pas solliciter notre conscience active, parce qu'elles correspondent aux scripts répétitifs des cadres sociaux et culturels qui régulent notre existence. Elles sont cependant constamment présentes et assurent le sentiment que nous avons de nousmêmes à travers le temps : elles nous relient à notre passé, celui de notre enfance comme celui du jour précédent, contribuant ainsi à construire la conscience de notre identité à travers le temps; elles nous permettent de structurer et d'interpréter ce que 
nous sommes en train de vivre, en le constituant précisément comme relevant de notre expérience propre ; elles jouent enfin un rôle de régulation, d'organisation, de projection du futur proche et lointain, en anticipant sur les heures, les années, la vie à venir. Dans ce sens, le biographique pourrait être défini, au côté d'autres formes de perception du vécu (cognitives, sensibles, affectives), comme une catégorie de l'expérience qui permet à l'individu, dans les conditions de son inscription socio-historique, d'intégrer, de structurer, d'interpréter les situations et les événements de son vécu. Cette activité de biographisation apparaît ainsi comme une herméneutique pratique, un cadre de structuration et de signification de l'expérience par lequel l'individu se donne une figure dans le temps, c'est-à-dire une histoire, qu'il rapporte à un soi-même.

\section{Formes sociohistoriques de la biographisation}

Les formes auxquelles les individus recourent pour biographier leur vie ne peuvent être abstraites des conditions historiques, sociales, culturelles et de l'ensemble des déterminations dans lesquelles se déroule leur existence. Les modèles, les schémas, les scripts, les figures auxquels les individus empruntent relèvent de constructions sociales et culturelles variables selon les époques et les sociétés. Il revient à la recherche biographique de donner toute sa dimension à une anthropologie historique des formes et des pratiques biographiques, en mettant en relation les modèles et les figures de biographisation disponibles dans telle société avec ses modes d'organisation politique, sociale, économique.

5 L'importance croissante du biographique dans les sociétés de la modernité est très directement liée aux mutations institutionnelles, économiques, technologiques que vivent ces sociétés depuis une trentaine d'années. Le passage de sociétés nationales, industrielles, centralisées, à des formes de société dont les organismes politiques, sociaux, économiques ont perdu de leur centralité, dont les institutions n'ont plus la même capacité d'intégration, s'accompagne de formes de socialisation faisant une part de plus en plus grande aux processus d'individualisation et de subjectivation: les individus de ces sociétés sont sommés de faire preuve de plus en plus d'initiative et d'autonomie et de trouver en eux-mêmes les ressources et les ressorts de leur conduite. Le sociologue américain Sennett (2000) rapporte qu'au début des années 1970 les ouvriers et les employés racontaient encore l'histoire de leur vie selon une structure linéaire continue et orientée, dont l'activité professionnelle (usine, bureau) constituait le motif organisateur et intégrateur : cette histoire était la leur et ils s'identifiaient à elle. Vingt années plus tard, la capacité de produire un récit unifié de la vie s'est considérablement réduite : à la place d'une histoire unique qui intègre tous les aspects de la vie sous un schème social et professionnel dominant, les informateurs délivrent des histoires plurielles et éclatées entre lesquelles ils peinent à établir de la continuité et de la permanence et qui demandent de leur part un travail biographique intense.

Le procès de subjectivation qui caractérise la société individualisée se traduit par une "culture héroïque du sujet » qui renvoie chacun à la construction et à la responsabilité de son propre parcours. Les travaux d'Ehrenberg ont bien mis en lumière l'injonction collective adressée à chaque individu de cultiver sa singularité, d'être le meilleur et le plus performant: "Chaque individu doit, dans son travail, ses loisirs ou sa vie affective, conduire sa vie comme un vrai professionnel de sa propre performance... Nous sommes désormais sommés de devenir les entrepreneurs de nos propres vies.» (1999, p. 16). La société de la modernité met chacun en demeure d'« avoir une vie ", une vie «à soi ", une vie 
qui lui soit propre : «La vie, écrit encore Ehrenberg, était vécue par la plupart des gens comme un destin collectif, elle est aujourd'hui une histoire personnelle." (1996, p. 18). L'injonction de la "vie propre» a son pendant immédiat dans un impératif de biographisation qui intime à chacun d'être « maitre et possesseur » de l'histoire de sa vie. Par-delà les séquentialisations et les standardisations collectives (âges de la vie, cursus scolaires, trajectoires professionnelles), les individus des sociétés postmodernes sont mis en demeure de procéder par eux-mêmes à la planification et à la gestion de leur parcours biographique.

7 Une des conséquences des mouvements de décentration et de désinstitutionnalisation qui affectent les sociétés postmodernes est en effet la séparation de l'acteur et du système, qui se traduit par la dilution et l'effacement de la notion de rôle, et la nécessité pour les individus de construire par eux-mêmes le sens de leur activité sociale. En quelque sorte, le niveau de la décision individuelle est remonté d'un cran dans le "pilotage » de l'activité sociale; de l'exécution d'actions programmées et de rôles établis, l'acteur social est passé aux «commandes " d'un ensemble de logiques possibles et de solutions alternatives, à partir desquelles il doit se frayer son propre itinéraire en orientant et en adaptant sa conduite (Dubet, 1995). Plus que par des rapports d'intégration et d'identification, l'activité sociale de l'individu postmoderne est régie par des rapports temporaires et réversibles d'inclusion dans des sous-systèmes auxquels il accède selon les secteurs de sa vie qui en relèvent; il est devenu l'homme pluriel décrit par Lahire (1998), qui n'est plus le représentant d'un groupe et de la logique sociale inhérente à ce groupe, mais le produit complexe d'expériences socialisatrices multiples. Le parcours biographique se définit dès lors comme le mouvement selon lequel un individu enchaîne et relie des profils d'insertion multiples à travers l'espace social : il est travailleur, consommateur, marié, contribuable, assuré, parent d'élève, membre d'une association sportive, électeur, etc., et il incorpore des modèles d'action différents et éventuellement contradictoires.

8 À la société complexe et foisonnante de l'ère postmoderne correspond une offre biographique infiniment plus ouverte et diversifiée, mais aussi moins hiérarchisée et cohérente que celle que proposaient des formes de société plus stables et plus centralisées. La pluralité des mondes sociaux confronte les individus à une multiplicité de curricula, parcours institués ou standards biographiques présents comme autant de trajectoires possibles dans la conscience individuelle. Dans la mesure où ces espaces sociaux ont perdu de leur centralité et ne s'organisent plus en un ensemble ordonné et hiérarchisé, les modèles biographiques de référence entrent en concurrence et ne font plus apparaître de façon distincte de schème biographique dominant. Si, dans les formes de société antérieures, la biographisation pouvait se réduire pour la grande majorité des individus à l'actualisation des patterns biographiques des groupes sociaux d'appartenance, dans la société postmoderne l'individu est contraint d'établir par luimême des liens et des modèles de coordination entre les "possibles biographiques " multiples et multiformes des mondes sociaux auxquels il participe. Chaque individu, tenu de se faire l'acteur biographique de sa propre vie, est ainsi renvoyé à un travail biographique intense pour tenter de rétablir de la continuité et de la cohérence entre des «histoires» plurielles, marquées par la dispersion, la fragmentation, la dissociation. Le paradoxe de la condition postmoderne est d'avoir remis à l'individu le soin de produire en lui-même le lien social que l'extrême différenciation et la complexité de la société d'aujourd'hui semblent avoir dénoué. Là où la sociologie classique parlait d'intériorisation ou d'incorporation des modèles sociaux et des habitus collectifs, il 
faudrait aujourd'hui parler d'autosocialisation pour désigner le processus selon lequel les membres des sociétés postmodernes travaillent à leur individuation en recherchant en eux-mêmes les ressources de leur socialisation.

\section{Travail biographique et socialisation}

La réflexivité biographique qui s'impose aux individus des sociétés modernes permet de mieux saisir ce qui est en jeu dans l'activité biographique : celle-ci se présente comme une forme de compréhension et de structuration de l'expérience et de l'action, s'exerçant de façon constante dans la relation de l'homme avec son vécu et avec son environnement social et historique. La rencontre de l'individuel et du social dans l'activité biographique relève d'un mode très particulier de perlaboration: selon leurs appartenances, leur âge, leurs catégories socio-professionnelles, leurs activités de socialité, les individus traversent successivement et quelquefois simultanément un grand nombre d'espaces sociaux et de champs institutionnels: famille, école et institutions de formation, marché du travail, profession et entreprise, institutions sociales et culturelles, associations et réseaux de socialité, etc. Or, ces données extérieures «objectives" ne sont pas perçues comme telles par l'individu, mais elles prennent pour lui l'aspect d'expériences qu'il rapporte à lui-même. Dans la conscience individuelle, les faits sociaux qui déterminent les situations, les interactions, les trajectoires, tout ce qui fait que la vie d'un individu est traversée de part en part par le social, relève de la logique des expériences accumulées et de la forme propre que ces expériences impriment au sentiment de soi-même et de son existence. L'individu ne peut saisir le social autrement que de manière auto-référentielle, en rapport avec son histoire et ses expériences, dans les formes de son «monde-de-vie »" , pour reprendre le concept développé par Schütz et Luckmann $(1975,1984)$ ou encore, selon la belle expression de Alheit et Dausien, en construisant "le monde intérieur du monde extérieur " (voir article dans le présent numéro). Les modes et les catégories selon lesquelles l'individu comprend et interprète de façon pratique la réalité sociale ne sont pas autre chose que les représentations qu'il tire de la généralisation de ses propres expériences. Pour l'individu, le monde social se constitue au fur et à mesure de ses expériences comme un ordre sensé d'actions. Les savoirs sociaux sont organisés dans la conscience individuelle sous forme de scripts d'action et de "plans de vie ». En particulier, les mondes sociaux auxquels participe l'individu sont appréhendés par lui sous la forme des programmes biographiques ou encore des "biographies typiques» dont ils sont porteurs. Chaque espace social (la famille, l'école, l'entreprise, etc.) spécifie ainsi des structures d'action et des profils biographiques qui font partie des savoirs transmis et sont actualisés et éprouvés dans l'expérience quotidienne.

9 Dans les sociétés de la modernité, la complexification et la diversification des mondes sociaux, les discontinuités des parcours personnels et professionnels, la coexistence dans la vie des individus de multiples réseaux de socialité multiplient ces biographies typiques, mais en même temps diluent leur emprise : les programmations biographiques anciennes perdent de leur centralité et de leur rigidité et les individus sont amenés à " choisir » entre des options biographiques multiples et à façonner par eux-mêmes le déroulement de leur vie. Devant établir par eux-mêmes et en eux-mêmes les modèles de coordination qui vont relier entre eux les différents espaces sociaux auxquels ils participent, les individus acquièrent une compétence réflexive qui les conduit à penser le déroulement de leur vie dans le champ de tension de leurs expériences et figurations 
biographiques et des contextes sociaux dans lesquels ils agissent: "Dans la société individualisée, écrit Beck, chacun doit apprendre à se percevoir comme le bureau d'étude de son propre curriculum » $(1986$, p. 217). Les individus sont amenés en particulier à calculer et à négocier le rapport de leur "biographie d'expérience » et des "biographies typiques» sous lesquelles se traduisent les normes et les prescriptions collectives. Le « cours de la vie » tend ainsi à s'instituer comme le lieu de processus de sélection, d'organisation, d'intégration par lesquels les individus s'autoinscrivent dans le monde social et contribuent à leur propre socialisation (Alheit \& Hoerning, 1989).

Ce que fait apparaitre en effet cette logique de subjectivation et d'appropriation biographique, c'est la dimension socialisatrice de l'activité biographique, le rôle qu'elle exerce dans la manière dont les individus se comprennent eux-mêmes et se structurent dans un rapport de co-élaboration de soi et du monde social. Qu'elles prennent la forme de scripts d'action, de constructions mentales, d'épisodes conversationnels, de récits de vie, les «histoires» que nous nous racontons à nous-mêmes et celles que nous adressons à autrui, loin de nous renvoyer à une intimité inaccessible, ont pour effet d'accorder notre espace-temps individuel à l'espace-temps social. Elles implicitent une connaissance et une pratique des contextes, des espaces sociaux, des modes d'action et de comportement; elles mettent en intrigue une rationalité et une opérativité sociales à laquelle nous sommes mêlés. Dans les opérations incessantes de biographisation auxquelles nous procédons, nous ne cessons en réalité de participer à la construction en nous et hors de nous de la réalité sociale, en la déclinant selon les multiples motifs et intrigues qui nous lient à elle. En quelque sorte, nous ne pouvons pas faire que les histoires selon lesquelles nous nous représentons, agissons, régulons, anticipons notre vie ne soient en même temps des histoires de société.

11 L'activité biographique accomplit ainsi une double et complémentaire opération de subjectivation $\mathrm{du}$ monde historique et social et de socialisation de l'expérience individuelle: elle est à la fois et indissociablement ce par quoi les individus se construisent comme êtres singuliers et ce par quoi ils se produisent comme êtres sociaux. La biographisation recouvre un ensemble d'opérations mentales, verbales, comportementales, par lesquelles les individus s'inscrivent subjectivement dans les temporalités historiques et sociales qui leur préexistent et les environnent, en s'appropriant les séquences, les programmes et les standards biographiques formalisés (curriculum scolaire, curriculum professionnel, mais aussi scripts d'action et scénarios) des mondes sociaux auxquels ils participent. Elle apparait en conséquence comme un processus essentiel de constitution de l'individu en société. Socialisant l'expérience individuelle dans des langages qui par définition sont des systèmes de signes partagés, elle donne une parole et une forme à ce qui sans elle en serait privé, elle fait passer dans l'univers des signes et des codes communs (représentations verbales, scénarios, récits, mais aussi gestes, comportements, attitudes) ce qui resterait proprement sans voix et sans figure, parce qu'incapable de sortir de la nébuleuse de l'indivis. La catégorie $\mathrm{du}$ biographique apparaît ainsi comme un principe d'organisation qui oriente et structure, sous la forme de langages partagés et transmissibles, l'expérience sociale quotidienne des individus. Selon les époques et les formes sociétales, les manifestations de ce processus de biographisation et l'intensité du travail biographique qui lui correspond varient en fonction du recours différencié que font les sociétés à la réflexivité individuelle et à ce champ privilégié de réflexivité que constitue la construction biographique. 


\section{Expérience biographique et rapport à la formation}

C'est sur le fond de cette réflexion qu'il faut revenir maintenant à ce qui fait l'objet de notre interrogation, le rapport biographique à la formation. La problématique envisagée n'est pas, on l'aura compris, celle du rapport des institutions formatives et de la «vie», ni ne se réduit (quoique devant forcément les recouper) à reprendre les termes du débat entre origine socioculturelle et accès au capital symbolique du savoir et de la culture. Elle consiste à prendre en compte, en la sortant de son cadre d'évidence, la dimension biographique de l'apprentissage et de la formation. Le rapport individuel à la formation comporte assurément un ensemble de données collectives (origine familiale, milieu social, formes instituées du cursus scolaire et des parcours professionnels), mais il s'inscrit également dans un espace et une temporalité propres au sujet : il n'est pas dissociable, d'une part, des représentations et des structures de savoir liées aux socialisations primaire et secondaire(s) de l'individu et aux «mondesde-vie» qu'elles ont contribué à construire, d'autre part de l'inscription des expériences de formation dans la dynamique d'une histoire individuelle où elles prennent leur place et leur signification. Il s'agit de reconnaître que la manière dont les individus biographient leurs expériences, et au premier chef la manière dont ils intègrent dans leurs constructions biographiques ce qu'ils font et ce qu'ils sont dans leur famille, à l'école, dans leur profession, en formation continue, est partie prenante du processus de formation.

\section{Expérience scolaire et formation adulte}

Un des apports importants du courant des « histoires de vie en formation » est d'avoir souligné la dimension expérientielle des apprentissages scolaires et de "l'école » en général et la manière dont cette expérience initiale est déterminante dans le rapport à la formation des individus adultes. Dominicé et son équipe à l'Université de Genève ont mené dans ce sens des travaux particulièrement significatifs, en interrogeant «la compétence d'apprendre de l'adulte en formation". Les conclusions de cette recherche montrent que le fondement "ontogénétique» du rapport à la formation et à la "compétence d'apprendre " adulte doit être recherché dans le rapport à l'école (Dominicé, 1999). Les biographies éducatives et les recueils de souvenirs scolaires font apparaître le poids de la scolarité initiale dans l'histoire de formation de leurs auteurs. Ces documents montrent que ce qui se noue pour chacun durant la période scolaire et qui est réactualisé dans le temps de la formation adulte, c'est une configuration de relations entre l'école, la famille, les groupes de pairs, le monde social en général. Cette configuration articule des formes de savoirs diversifiés et vécus souvent sur un mode oppositif ou antinomique (savoirs de l'école versus savoirs de la vie), dont l'équilibre ou le déséquilibre marque pour longtemps l'histoire de formation du sujet. Pour que la formation de l'adulte ait un sens, il faut qu'elle puisse être resituée dans cette histoire et que l'adulte soit en mesure de prêter une signification aux premières étapes de sa formation à l'école. Cette démarche passe par une réévaluation des acquis scolaires considérés non pour eux-mêmes et en eux-mêmes mais dans leur rapport au parcours de formation: «Le savoir de l'école, écrit Dominicé, prend sens dans les expériences de la vie» (Dominicé, 1999, p. 14). 
Cette réévaluation de la formation scolaire suppose que l'on reconnaisse que les acquis de l'école, loin de se limiter à des savoirs disciplinaires et formalisés, sont des alliages de savoirs qui comportent également des savoirs expérientiels trouvant leur traduction dans des comportements, des modes de relations, des émotions et des sentiments, des formes de pensée et de raisonnement. L'école elle-même, - en tant qu'espace physique, social, et en tant qu'espace cognitif, qu'espace où on acquiert des connaissances -, représente un champ particulier d'expériences correspondant à des acquis existentiels déterminants pour la formation à venir. Le regard globalement négatif porté sur le vécu scolaire vient en partie de ce que le système école lui-même méconnaît, disqualifie, occulte pour une large part les savoirs expérientiels dont il est le terreau, au profit des savoirs formalisés qu'il pense seuls susceptibles d'être mesurés, sanctionnés, validés. Les documents biographiques en témoignent amplement : ce que les adultes en formation retiennent de l'école, ce qu'ils désignent de leur temps scolaire comme étant significatif pour leur parcours de formation, ce sont, beaucoup plus que des contenus de connaissance, les expériences relationnelles, affectives, sociales, dont l'école a été pour eux le champ et dont la polarité émotionnelle a marqué leur rapport à l'apprentissage et au savoir.

\section{L'école comme expérience biographique}

Cette prégnance de l'école dans la compétence d'apprendre de l'âge adulte invite à interroger l'expérience biographique de l'école elle-même. Comment les élèves rencontrent-ils le monde social et culturel de l'école? Quel sens et quelle forme lui donnent-ils dans leur histoire individuelle et dans les figurations qu'ils ont d'euxmêmes? Comment investissent-ils biographiquement le domaine propre d'activité et de production de l'institution scolaire, la transmission-appropriation des savoirs?

La manière dont les élèves vivent, se représentent et font signifier l'école et ce qu'ils y font ne peut manquer de rencontrer, sous des angles et sur des modes divers, la façon dont ils se racontent eux-mêmes et ce qu'ils se racontent sur eux-mêmes. Cette « rencontre » prend plusieurs aspects complémentaires : d'abord, l'école, en tant que milieu social organisé et spécifié dans ses tâches et ses fonctions, entre en relation avec une histoire individuelle qui a son origine avant elle et qui se poursuit après elle, histoire qui se décline en un faisceau de déterminations, de représentations, de projections collectives et individuelles; par ailleurs, l'école est elle-même porteuse d'histoire: composante de l'histoire présente des élèves, elle est aussi composante de leur histoire à venir et vient interférer, par la sélection qu'elle opère, les orientations qu'elle détermine, les modèles de parcours et de finalisation qu'elle propose, dans les attentes et les projets des élèves et de leurs familles ; enfin, dans son activité propre de transmission-acquisition de savoirs, l'école définit entre les individus qu'elle réunit, adultes et non-adultes, des positions et des rôles spécifiés par l'accomplissement de tâches d'enseignement-apprentissage faisant l'objet d'évaluation en termes de réussite ou d'échec. Sous ces différents aspects qui composent l'expérience subjective des élèves, l'école est au centre de multiples jeux de représentations dans la manière dont les élèves construisent pour eux-mêmes et pour les autres leur histoire et leur devenir. L'espace scolaire apparaît ainsi pour les élèves comme un lieu fort de biographisation, à la fois par la place et le sens qu'ils donnent à l'école dans leurs constructions 
biographiques et par les modèles de biographisation auxquels l'école les confronte pour le présent de leur vie d'élèves comme pour le futur de leur vie d'adultes.

Tant dans les sciences sociales que dans les sciences de l'éducation, l'attention portée à l'expérience scolaire et les recherches qui lui sont consacrées sont de date récente. Elles ont pour origine une interrogation sur l'échec scolaire et la remise en cause de points de vue disciplinaires exclusifs ramenant la réussite ou l'échec à l'école à des facteurs explicatifs uniques comme le profil psychologique, le développement cognitif ou le handicap socioculturel, et elles s'inscrivent explicitement ou implicitement dans le cadre d'une théorie de l'acteur considérant les individus-élèves dans la globalité de leurs inscriptions, de leurs actions et de leurs représentations. Parmi les études les plus significatives, on retiendra en particulier celles de Dubet sur l'expérience lycéenne et les travaux menés par Charlot, Bautier et Rochex dans le cadre de l'équipe de recherche ESCOL de l'Université Paris-8 autour de la notion de "rapport au savoir " (Bautier, 1995 ; Bautier \& Rochex, 1998 ; Charlot, 1997, 1999 ; Charlot, Bautier \& Rochex, 1992 ; Rochex, 1995).

L'enquête sociologique de Dubet (1991) sur la diversité de l'expérience lycéenne met en avant les modalités et les attitudes selon lesquelles les lycéens se positionnent et se différencient face à l'école et aux apprentissages scolaires. Elle montre que ces apprentissages s'inscrivent et prennent sens dans un rapport d'identité et d'expérience à l'univers social et affectif des élèves, univers à la fois extérieur à l'enceinte scolaire et intérieur à l'école en tant qu'elle constitue elle-même un milieu social producteur de rôles, de valeurs, d'images de soi et de trajectoires. Dubet resitue l'expérience scolaire dans le cadre des fonctions du système éducatif : fonction de formation (transmission de la culture), fonction de sélection (classement des élèves selon leurs compétences scolaires) et fonction d'intégration (capacité de l'école à reconnaître «la communauté et la vie juvéniles»). Venant s'inscrire dans le jeu de relations entre ces trois fonctions, l'expérience lycéenne est décrite selon trois dimensions. La première est celle du projet de l'élève qui définit l'utilité des études en les inscrivant dans un parcours, dans la perspective d'une réalisation sociale et professionnelle plus ou moins définie. La seconde est celle de la formation du sujet et touche au sens subjectif que les élèves donnent aux disciplines qui leur sont enseignées, aux savoirs qu'ils acquièrent, aux relations pédagogiques avec les enseignants, mais aussi à l'espace de l'école comme lieu d'accueil et d'expression de la vie juvénile (groupes d'affinités, discussions entre pairs, relations amicales et amoureuses). La troisième dimension est celle de la stratégie qui se rapporte à la manière dont les élèves gèrent les positions qu'ils occupent dans l'espace réglementé de l'école et sur l'échelle où les situent leurs performances scolaires, dont ils développent pour ce faire des stratégies d'adaptation et d'intégration, calculent leurs investissements et leurs intérêts et mènent de façon plus ou moins habile leur «métier d'élève ».

\section{Formes et enjeux du rapport au savoir}

La recherche de l'équipe ESCOL a montré le rôle majeur que joue le « rapport au savoir " dans les processus de différenciation scolaire et dans la production de la réussite et de l'échec. Le rapport au savoir est fondé sur le sens que les élèves donnent aux objets d'apprentissage et aux situations scolaires, à la façon dont ils font signifier leur fréquentation de l'école, les tâches qu'ils sont amenés à y faire, les savoirs qu'ils y construisent : "Tous les élèves donnent et construisent - pour une part à leur insu-un sens 
aux objets d'apprentissage et aux situations scolaires, mais ce sens est différent chez les uns et les autres, et il peut être de nature à favoriser ou, au contraire, à gêner l'appropriation des savoirs » (Bautier \& Rochex, 1998, p. 35).

Le rapport au savoir peut être investi selon deux registres principaux qui correspondent à deux positions différenciées face à l'école et aux apprentissages. Le registre identitaire inscrit le rapport au savoir dans l'histoire du sujet et dans les représentations qu'il a de lui-même : À quelles attentes biographiques et identitaires répondent les apprentissages et les savoirs de l'école? Comment sont-ils liés dans les représentations de l'élève à la vie qu'il veut mener, au métier qu'il veut faire, etc. ? Le registre épistémique définit le rapport que l'élève entretient avec l'apprendre et le savoir considérés en eux-mêmes: Qu'est-ce qu'apprendre? Que fait-on quand on apprend ? Quel intérêt et quel sens ont les savoirs en eux-mêmes ? (Charlot et al., 1992, pp. 31-32). Registre identitaire et registre épistémique ne sont pas exclusifs l'un de l'autre, leur articulation et leur combinaison varient d'un élève à l'autre et engagent des logiques différenciées dans les processus d'appropriation des savoirs et dans la façon dont les élèves les font signifier. À partir d'entretiens et de bilans de savoirs menés auprès d'élèves de différents collèges de la région parisienne, les membres de l'équipe ESCOL ont pu donner des représentations idéales-typiques de ces formes différenciées de rapport au savoir: le premier idéaltype est celui de l'élève qui assimile l'apprentissage aux formes extérieures du «métier d'élève » et du «travail » scolaire : "Apprendre à l'école, c'est écouter les professeurs, lever la main avant de parler, faire ses devoirs, apprendre ses leçons, apprendre ce qu'il faut savoir quand on est collégien, aller aux cours qui se succèdent tout au long de la semaine » (Charlot et al., 1992, p. 148). Dans ce cas, apprendre ne signifie pas s'approprier les savoirs, mais se conformer à un ordre et à un code scolaires, maîtriser les situations de la vie scolaire vécue comme un des espaces de la vie quotidienne. Ce que l'on fait à l'école n'a pas de sens en soi-même, l'école n'apparaît «utile » que dans la perspective d'un métier, d'un avenir auxquels elle permettrait d'accéder. Pour le deuxième idéaltype, l'école n'est pas non plus le lieu d'apprentissage de savoirs objectivés, mais elle représente cependant un espace où l'élève apprend à maîtriser les situations et à se donner les moyens de cette maitrise : "... maîtriser une situation, c'est désormais "s'exprimer", "s'organiser", "penser", "réfléchir", et non plus simplement écouter le professeur et faire le programme. Apprendre, ce n'est plus se rendre capable d'agir en situation, mais sur la situation " (Charlot et al., 1992, p. 149). Chez certains élèves, en particulier les filles, le processus d'appropriation ainsi mis en œuvre peut s'étendre bien au-delà de la sphère scolaire et se présenter comme une réflexion et un projet de maîtrise sur « la vie ", « le monde ", « les gens ». Le troisième idéaltype correspond à l'élève qui objective les savoirs et leur donne du sens en eux-mêmes : «Apprendre, c'est apprendre des choses précises, énonçables, c'est s'approprier des objets intellectuels. L'objet de l'apprentissage n'est pas la situation... mais un contenu intellectuel spécifique (le cosinus, la vie de nos ancêtres, les composants de ce qui nous entoure, etc.)" (Charlot et al., 1992, p. 150). Cet idéaltype répond au profil du «bon» élève, qui ne réduit pas l'apprentissage à l'effectuation de gestes et de comportements scolaires, qui n'évalue pas les savoirs à la mesure de leur utilité immédiate ou à venir, mais qui les considère comme des constructions intellectuelles trouvant leur valeur en elles-mêmes.

17 Ce clivage entre élèves fixés sur la tâche, élèves fixés sur la situation et élèves s'appropriant des savoirs - et le malentendu qu'il révèle chez les premiers et en partie chez les seconds sur la nature et le sens des activités scolaires - est en corrélation avec 
les différences de conception et d'interprétation que les élèves donnent à la scolarisation, objet possible d'un malentendu plus général sur l'école. La centration sur la tâche va souvent de pair avec une représentation de la scolarité vécue comme une succession de niveaux à franchir, de classes à "passer ", dans une logique décrite comme une logique de «cheminement » et d'effectuation du "métier d'élève ». Le cursus scolaire est vécu comme la course d'obstacles consistant pour l'élève à accomplir les gestes et les rituels que l'on attend de lui (obéir aux consignes, observer les règles de comportement) pour obtenir cette forme de reconnaissance qui seule donne un sens à sa présence à l'école : passer dans la classe supérieure. Cette attitude vis-à-vis de la scolarité s'accompagne souvent d'une représentation "utilitariste» de l'école: de même que le « métier d'élève » consiste à faire (est conçu comme consistant à faire) ce qu'il faut (ce que l'on croit devoir faire) pour gravir les échelons du parcours scolaire, de même l'école est conçue comme une échelle sociale destinée à donner un métier et à assurer une place dans la société, sans que soit posée la question de la nature de l'activité intellectuelle et des contenus de savoir que requiert l'accession à tel métier ou à telle position sociale. À l'inverse, les élèves qui vivent leur scolarité comme un temps d'apprentissage et d'appropriation de savoirs donnent à leur activité scolaire une signification intrinsèque, en reconnaissent et en valorisent la dimension cognitive et trouvent dans la construction des savoirs des occasions et des ressources d'élaboration personnelle.

\section{Mondes sociaux, monde de l'école et travail biographique}

Les registres du rapport au savoir tels qu'ils sont vécus et interprétés par les élèves, les représentations de l'école et du cursus scolaire qui les accompagnent, engagent et distinguent de fait des modes différenciés de rapport à soi-même et de biographisation. Pas plus que chez les adultes, le rapport au savoir des élèves n'est un rapport biographiquement désinvesti : il engage des figures de soi qui ont leur répondant dans différents « lieux » réels ou symboliques. Ces formes de biographisation du rapport au savoir illustrent toutes un même propos : le savoir prend son sens dans un lieu, dans une situation, dans un rapport à soi-même, aux autres et au monde; il s'inscrit dans un réseau complexe de socialité qui embrasse l'ensemble des espaces sociaux auxquels participe l'élève. Comment se rencontrent apprentissages scolaires et apprentissages de soi dans le monde de l'école ? À quelles formes de biographisation recourent les élèves pour tenter de satisfaire à la fois au projet de l'école et à la construction de leur figure de soi ?

La figure de soi des enfants et des adolescents qu'accueille l'école se construit dans une pluralité de mondes sociaux parmi lesquels l'école n'occupe pas forcément une position prioritaire. Les mondes sociaux auxquels participent les jeunes dans les sociétés postmodernes sont beaucoup plus nombreux et complexes qu'ils ne l'étaient dans les formes de sociétés antérieures. Dans la plupart des cas, le monde-de-vie juvénile se construit aujourd'hui sous des formes mouvantes et instables, qui exigent de la part des individus un travail biographique ${ }^{2}$ intense. Celui-ci permet à la fois de métaboliser des expériences contradictoires ou dissociatives et de modifier dans le sens d'une nouvelle figuration de soi les représentations qui orientent les individus dans leur appréhension des situations et des personnes. Cette activité de mise en cohérence ne se fait pas sans heurts ni ruptures. Les mondes sociaux auxquels les jeunes participent présentent parfois entre eux de telles divergences sociales et culturelles qu'ils doivent fournir un 
réel effort pour les relier les uns aux autres et pour relier les expériences qu'ils y vivent. Ces mondes sociaux pluriels induisent des figures de soi divergentes et parfois même contradictoires ${ }^{3}$ produisant des effets de discontinuité biographique. Alheit a suggéré le terme de patchwork biographique pour désigner cette segmentation de l'expérience à partir de laquelle les individus tentent de composer une image unitaire d'eux-mêmes et du cours de leur vie (Alheit, 1997).

L'appartenance à divers mondes sociaux au cours de l'enfance puis de l'adolescence conduit à incorporer des règles, des habitus, des valeurs symboliques variés et parfois contradictoires. Chaque situation est l'occasion d'une expérience nouvelle par laquelle le jeune réajuste son monde-de-vie antérieur. Une fois métabolisées, ces expériences deviennent des ressources biographiques qui constituent la matrice à travers laquelle de nouvelles expériences biographiques sont identifiées et constituées. Ce processus permanent peut s'accomplir dans une certaine fluidité mais certains épisodes de transformation ou de rupture de l'expérience donnent lieu à des tensions qui requièrent une importante activité de biographisation. Il faut trouver la forme d'inscription biographique de nouvelles expériences qui bousculent la configuration antérieure et qui amènent à une transformation de la perception du monde, des autres et de soi-même. Le monde réglé et programmé de l'école offre peu de place (en espace et en temps) à ces processus de configuration/déconfiguration/reconfiguration, et c'est sans doute à l'école que se font le plus vivement ressentir les difficultés éprouvées à réorganiser le monde-de-vie et à réajuster le projet de soi. En même temps, la période de l'école, et plus spécialement celle qui correspond à l'adolescence, est la plus inventive en figures et figurations de $\operatorname{soi}^{4}$ et la préoccupation du projet de soi y prend une intensité particulière. Les figures de soi que l'élève construit dans les autres mondes sociaux continuent à exister à l'école et l'élève doit sans cesse dégager leurs points d'intersection avec celles qu'il développe dans le monde de l'école. Ces figures de soi peuvent entrer en opposition, n'avoir plus d'articulation suffisante entre elles, ou se réduire à une figure dominante requérant une grande mobilisation biographique. L'école peut ne plus avoir sa place dans le travail biographique intense que doit alors fournir l'élève : il perd pied, « décroche » momentanément ou durablement. Son projet de soi n'est plus en lien avec le projet que lui propose l'école.

Les figures de lui-même que l'élève introduit et expérimente dans l'espace scolaire se trouvent confrontées au projet que l'école a pour lui et qui se traduit par un cursus d'études, par des programmes et des contenus de savoirs, par la prescription d'attitudes et de conduites qui ont rapport à la fois à l'acquisition des objets propres de l'école et aux relations qui doivent prévaloir entre ses usagers, non-adultes et adultes. Le projet de l'école est le reflet d'un état de société, des savoirs et des valeurs en lesquelles une collectivité se reconnaît et qu'elle institue comme modèles de référence pour ses membres les plus jeunes. Au-delà de ses aspects explicites en termes de cursus à parcourir et de savoirs à acquérir, ce projet dessine un certain type de relations de l'individu à la collectivité et de l'individu à soi-même, type de relations qui reproduisent le régime général des rapports sociaux dans l'espace social environnant. L'école institue ainsi des trajectoires idéales-typiques qui correspondent à des figures également idéales-typiques de la réussite (scolaire et sociale), pour lesquelles certains savoirs disciplinaires (aujourd'hui les mathématiques et les sciences dures) jouent un rôle indicatif et sélectif majeur. Le rapport de l'école au savoir est loin de se jouer sur le seul plan épistémique : il inclut une dimension fonctionnelle et structurelle de sélection 
qui passe par des procédures de filiarisation, d'évaluation, de notation, de classement, procédures qui composent le quotidien de l'expérience scolaire des élèves. Le rapport que l'école institue au savoir et à l'apprendre est évalué en termes de réussite et d'échec et ce rapport se traduit par des modes d'étiquetage codifiés et restrictifs de ce que font et de ce que sont les « individus-élèves » et par des typisations construites sur un critère quasi exclusif de performance dans les apprentissages scolaires. De ce point de vue, l'artefact « élève » est une construction fonctionnelle et maniable qui se décline selon les étapes instituées du cursus scolaire (passage d'un secteur d'enseignement à un autre, passage de classe en classe) et sur le mode d'un système d'attribution de qualité (les appréciations du bulletin scolaire) très fortement articulé sur les "résultats " obtenus dans les apprentissages. Malgré son caractère artificiel, la représentation biographique liée à cette construction "élève » est extrêmement prégnante, elle s'impose aux élèves ainsi qu'aux professeurs comme la règle d'un système auquel il est très difficile de se soustraire, et une grande partie de l'expérience scolaire consiste à négocier pour soi-même et pour les autres (groupes de pairs ou adultes) les étiquetages et typisations de l'école et les constructions biographiques personnelles.

Parce qu'elle correspond à une première étape de socialisation secondaire et parce qu'elle se traduit en structures d'action et en modèles de conduite fortement marqués, l'école rend ainsi particulièrement sensible la confrontation entre la "biographie d'expérience " des enfants et des adolescents qu'elle accueille et les "biographies typiques » dont elle est porteuse, de la première et plus prégnante d'entre elles, celle de l'«élève », aux modèles de parcours intellectuels, professionnels et sociaux qu'elle implicite.

\section{L'expérience biographique comme structure d'apprentissage}

C'est cette même confrontation entre «biographie d'expérience» et «biographies typiques » qui est réactualisée et réactivée dans chacun des épisodes de la formation. Cette confrontation ne met pas seulement aux prises les biographies individuelles dans leur infinie singularité et diversité et les standards biographiques que véhiculent les institutions formatives : elle engage le mode de constitution de l'expérience biographique et la manière dont celle-ci structure l'appréhension et la construction des « objets » de la formation, qu'il s'agisse de savoirs formalisés, de savoir-faire procéduraux, de compétences multidimensionnelles, etc. L'expression expérience biographique peut sembler tautologique: quelle que soit sa nature (intellectuelle, affective, esthétique, religieuse, etc.), toute expérience est biographique, dans la mesure où elle est constituée précisément par l'inscription d'une situation, d'un événement, d'un " objet » dans la vie d'un individu et dans les représentations qu'il s'en construit. L'expression se justifie cependant si l'on considère la logique biographique dont elle relève. C'est cette logique de construction biographique qui fait que l'on ne peut en rien comparer la manière dont un individu s'approprie des connaissances et la manière dont est alimentée une banque de données dans un système informatique, mais que l'on peut en revanche rapprocher, sur le plan des processus mis en œuvre, une expérience cognitive et une expérience affective.

La constitution biographique de l'expérience transparaît dans la distinction que fait le langage courant entre avoir de l'expérience et faire une expérience : la première expression désigne l'expérience comme un ensemble de compétences et de "savoirs" de tous ordres à notre disposition: c'est l'expérience de l'«homme expérimenté», de 
l'« homme d'expérience ", mais aussi, d'une façon unanimement partagée, celle qui est constituée progressivement tout au long de l'évolution individuelle (tout individu humain acquiert de l'expérience); la seconde expression désigne l'expérience comme ce qui survient dans le présent du cours de la vie : cette expérience qui survient lorsque «nous faisons une expérience » se présente au premier regard comme l'incidence dans notre vie d'un événement inattendu, d'une situation surprenante; elle est ce qui échappe à nos routines (comportements, savoirs) et ce qui précisément n'entre pas dans le champ de l'expérience acquise et des savoirs disponibles qui la constituent. L'expérience en tant qu'événement survenant consiste d'abord dans l'impossibilité de mettre en rapport ce qui nous arrive et la réserve de savoirs que nous avons constituée au fil de notre vie et de nos socialisations. Pour que fasse expérience ce qui nous est étranger et inconnu, il faut que nous le soumettions à des opérations d'étiquetage, de classement, de typisation, qui vont venir l'intégrer et le ranger dans la réserve de nos savoirs disponibles. Sous des rapports variables selon la nature des nouveaux savoirs intégrés, cette réserve de savoirs va s'en trouver modifiée et reconfigurée ${ }^{5}$.

C'est selon cette logique de construction biographique que la situation ou l'objet nouveau vient trouver (ou non) sa place et sa forme particulière au sein des expériences antérieures de formation et s'intégrer à la structure de connaissance que constitue l'expérience constituée. Pour que le sujet se les approprie, les objets de la formation doivent faire l'objet d'une interprétation et d'une intégration dans les systèmes de connaissances ou de compétences antérieurs des formés (qui ne sont pas identiques entre eux, qui sont différents de celui du formateur et qui ne reproduisent pas le système objectivé et formalisé du domaine de savoir ou de compétence concerné) ${ }^{6}$. Tout objet nouveau d'apprentissage engage ainsi un procès unique (propre à chaque individu, et unique dans son histoire d'apprentissage) d'appropriation et de reconfiguration de l'ensemble construit des connaissances et compétences acquises. Pour rendre compte de cette dimension qui fait de chaque épisode de formation une expérience biographique, Alheit et Dausien proposent dans ce même numéro le concept d'« apprentissage biographique» (biographisches Lernen) : "L'apprentissage [apparaît] comme trans-formation d'expériences, de structures de savoirs et d'action en une configuration biographique particulière... Les domaines de l'expérience que les institutions et la société séparent et spécialisent sont intégrés selon un processus biographique de concrétion de l'expérience et composent une figure de sens particulière " ${ }^{7}$.

Dans les procès de formation, les savoirs et les compétences se trouvent ainsi défaits et recomposés selon une logique de l'expérience qui ne respecte pas les découpages disciplinaires ou instrumentaux, mais les soumet à un processus d'appropriation de type biographique. Si chaque apprentissage relève d'une construction particulière de l'expérience, la structure de connaissance à laquelle il vient s'intégrer a par conséquent sa propre histoire et renvoie à une biographie de l'expérience individuelle. En ce sens, l'apprentissage peut être défini comme une activité autoréférentielle: le savoir nouveau rencontré dans l'histoire d'apprentissage doit d'abord être traduit dans le code des savoirs acquis avant de développer son efficacité. Ce code est lui-même le résultat unique d'une concrétion d'expériences d'apprentissage qui définit précisément la biographie d'apprentissage. Le principe d'autoréférentialité biographique qui définit la structure d'apprentissage est cependant lié aux langages et aux significations du monde social environnant, dans la mesure où les sollicitations d'apprentissage et les savoirs 
qui leur correspondent ont leur origine dans le monde social. Les procès d'apprentissage sont ainsi des constructions biographiques socialement inscrites.

\section{Conclusion}

Les notions d'expérience, de rapport au savoir, de biographie d'apprentissage mettent toutes en avant le rôle central de l'apprenant dans le procès de formation et dans l'appropriation des savoirs: l'apprenant est l'auteur (l'origine en termes de désir, d'" autorisation ", de vouloir), l'acteur (l'agent en termes de moyens, de travail, de production) et le bénéficiaire (la visée en termes d'intention et de projet) du processus d'apprentissage et de formation. Elles contribuent également à une meilleure compréhension de l'histoire de formation des individus entre les déterminismes sociaux et les trajectoires individuelles. Si les apprentissages et les parcours de formation sont structurés par les cadres sociaux et institutionnels dans lesquels ils se déroulent, s'ils sont modélisés par les représentations collectives que s'incorporent les individus, ils s'inscrivent cependant toujours dans des biographies qui ne sont réductibles ni au mécanisme exclusif de contraintes sociales extérieures ni à une instance purement subjective et tirent précisément leur singularité d'un jeu unique d'interrelations entre modèles sociaux et expériences individuelles, entre déterminations sociohistoriques et histoire personnelle, entre prévisibilité biographique et projet de soi. Tout procès de formation, structuré ou non, intentionnel ou non, est un acte socialement situé et socialement construit, mais il n'y a de formation qu'inscrite dans la singularité d'une biographie. La formation, de quelque nature qu'elle soit, est toujours la mise en œuvre et le résultat du travail de réflexivité et de subjectivation qu'elle engage chez la personne sur qui elle s'exerce et qui s'en approprie la visée en lui donnant place dans son propre projet de soi. Mais à l'instar de ce qui se passe dans le travail biographique, ce processus d'appropriation est en même temps un processus de socialisation, puisqu'il consiste à partager en les faisant siens des conduites et des valeurs communes, des représentations et des conceptions communes, des savoirs et des savoir-faire communs, c'est-à-dire à trouver sa place dans le lieu commun du lien social. Les procès de formation sont des cas particuliers de biographisation qui consistent à faire entrer dans la figure et le projet de soi les visées et les valeurs qu'une collectivité, une institution, un groupe social tiennent pour représentatives de ce qu'ils sont et qu'ils constituent en modèles et en projets éducatifs. Au sens que nous avons donné à ces notions, l'activité biographique est le moteur de ce processus d'appropriation de la formation et la biographie l'horizon sur lequel viennent s'inscrire les projets éducatifs spécifiques dont sont porteurs les mondes sociaux. Qu'il soit enfant, adolescent ou adulte, l'acteur biographique intègre les épisodes particuliers d'apprentissage et de formation dans le mouvement général de sa propre biographisation, c'est-à-dire du développement de son projet de soi au sein de l'espace social. 


\section{BIBLIOGRAPHIE}

Alheit, P. (1997). Patchworking als moderne biographische Konstruktionsleistung. Bemerkungen zur theoretischen Bedeutung einer Biographieorientierung in der Erwachsenenbildung (Le patchworking comme compétence de construction biographique de la modernité). In K. DerichsKunstmann, P. Faulstich, \& R. Tippelt (Eds.), Enttraditionalisierung der Erwachsenenbildung (Beiheft zum Report). Frankfurt am Main.

Alheit, P., \& Dausien, B. (2002). Bildungsprozesse über die Lebensspanne und lebenslanges Lernen (Les processus de formation et l'apprentissage tout au long de la vie). In Hrsg von R. Tippelt (Ed.), Handbuch Bildungsforschung (p. 217).

Alheit, P., \& Dausien, B. (2004). Les processus de formation et l'apprentissage tout au long de la vie ». L'Orientation Scolaire et Professionnelle, 33, 4 (Travail biographique, construction de soi et formation), Delory-Momberger, Ch. (coord.).

Alheit, P., \& Hoerning, E. (Hrsg) (1989). Biographisches Wissen. Beiträge zu einer Theorie lebensgeschichtlicher Erfahrung (Savoir biographique. Contributions à une théorie de l'expérience biographique). Frankfurt/New York : Campus.

Bautier, E. (1995). Pratiques langagières, pratiques sociales. Paris : L'Harmattan.

Bautier, E., \& Rochex, J.-Y. (1998). L'expérience scolaire des nouveaux lycéens. Démocratisation ou massification? Paris : Armand Colin,

Beck, U. (1986). Risikogesellschaft. Auf dem Weg in eine andere Moderne (La Société du risque. Sur le chemin d'une autre modernité). Frankfurt/M : Suhrkamp.

Beillerot, J. (1982). La Société pédagogique. Paris : P.U.F.

Charlot, B. (1997). Du rapport au savoir. Éléments pour une théorie. Paris : Anthropos.

Charlot, B. (1999). Le rapport au savoir en milieu populaire. Paris : Anthropos.

Charlot, B., Bautier, E., \& Rochex, J.-Y. (1992). École et savoir dans les banlieues... et ailleurs. Paris : Armand Colin.

Delory-Momberger, Ch. (2003). Biographie et éducation. Figures de l'individu-projet. Paris : Anthropos.

Dominicé, P. (1999). La compétence d'apprendre à l'âge adulte : lectures biographiques des acquis de la scolarité. Cahiers de la section des sciences de l'éducation, 87 (Les origines biographiques de la compétence d'apprendre). Université de Genève.

Dubet, F. (1991). Les Lycéens. Paris : Le Seuil.

Dubet, F. (1995). Sociologie de l'expérience. Paris : Le Seuil.

Ehrenberg, A. (1996). L'individu incertain. Paris : Hachette Littérature.

Ehrenberg, A. (1999). Le culte de la performance. Paris : Hachette Littérature.

Kraull, M., \& Marotzki, W. (2002). Biographische Arbeit. Oplade : Leske+Budrich.

Lahire, B. (1998). L'Homme pluriel. Les ressorts de l'action. Paris : Nathan.

Ricœur, P. (1983). Temps et récit I. Paris : Le Seuil.

Rochex, J.-Y. (1995). Le sens de l'expérience scolaire. Paris : P.U.F. 
Schapp, W. (1992). Empêtrés dans des histoires. L'être de l'homme et de la chose (Traduction : Jean Greisch). Paris : Cerf.

Schütz, A., \& Luckmann, Th. (1 $1^{\mathrm{er}}$ vol. $1975,2^{\mathrm{e}}$ vol. 1984). Strukturen der Lebenswelt. Frankfurt am Main : Suhrkamp.

Sennett, R. (2000). Le travail sans qualités. Les conséquences humaines de la flexibilité. Paris : Albin Michel.

\section{NOTES}

1. Nous utilisons cette expression, empruntée à la sociologie allemande (Lebenswelt), pour désigner, non la réalité de l'environnement vécu, mais le système construit des représentations biographiques. Dans la sociologie de la connaissance développée par Alfred Schütz, le monde-devie (Lebenswelt) est constitué par la réserve d'expériences et de savoirs qui nous sont transmis par notre milieu familial et social d'origine et par ceux que nous constituons par nous-mêmes lors de nos socialisations successives. Cette réserve de savoirs disponibles forme un univers symbolique de référence dans les situations de la vie quotidienne : «L'homme, dans son quotidien, dispose à tout moment d'une réserve de connaissances qu'il utilise comme schème d'interprétation de ses expériences passées et présentes et qui détermine aussi ses anticipations sur les choses à venir. » A. Schütz, Le Chercheur et le quotidien, Paris, Méridiens Kincksiech, 1987, p. 203.

2. La notion de travail biographique désigne l'activité produite par l'individu pour donner une cohérence et un sens aux événements de sa vie. Elle trouve son origine dans des travaux sociologiques sur la flexibilité du travail dans les sociétés postindustrielles et sur son incidence sur les processus de biographisation (Sennet, 2000 ; dans le domaine de la formation, Kraull \& Marotzki, 2002).

3. C'est par exemple le cas de jeunes d'âge scolaire devant assurer des "petits boulots » pour apporter une aide financière à leur famille. Par ailleurs, si les parents sont illettrés et parlent mal le français, les jeunes auront la charge des démarches administratives. Il est clair que ces jeunes se retrouvent dans des projets de soi et des images de soi fortement opposés : ils sont élèves à l'école, co-responsables de la famille, «protecteurs » de leurs parents dans le pays d'accueil.

4. La distinction que nous faisons entre figure de soi et figuration de soi est la suivante : la figure de soi renvoie à une image intérieure de soi-même construite à l'intersection du sentiment (du ressenti) et de l'expérience que l'on a de soi, et des « langages » à disposition dans l'espace social ; l'ensemble des figures de soi s'organise de façon dynamique et orientée pour former le projet de soi. Quant au terme figuration de soi, il est susceptible de deux acceptions: soit il désigne l'opération de mise en figure, soit il renvoie aux manifestations extérieures (en termes de postures, d'attitudes) que prend la figure de soi.

5. Cette description de l'expérience et de son fonctionnement a été développée en particulier par A.Schütz et T.Luckmann dans Strukturen der Lebenswelt (Structures du monde-de-vie) Frankfurt/Main, Suhrkamp, 1975, 1984. Je donne une description détaillée de la structure d'expérience et de connaissance théorisée par A.Schütz sous la notion de "monde-de-vie» (Lebenswelt) dans Biographie et éducation. Figures de l'individu-projet, pp. 95-99.

6. Il règne un certain flou notionnel dans l'usage qui est fait des termes "savoir " et « connaissance ». Si le respect des traductions et les expressions consacrées ne rendaient la chose quelquefois difficile, je me rallierais volontiers à la position suivante: le savoir est ce qu'une société reconnaît de façon instituée, ce que la science établit et que l'école enseigne; la « connaissance » est à la fois le processus par lequel l'individu s'approprie des savoirs et le produit de cette appropriation comme composante du système de connaissances individuel. 
7. Alheit, P. \& Dausien, B. « Bildungsprozesse über die Lebensspanne und lebenslanges Lernen » (Les processus de formation et l'apprentissage tout au long de la vie) in Handbuch Bildungsforschung (Hrsg von R. Tippelt), 2002, p. 217.

\section{RÉSUMÉS}

À partir d'une analyse du biographique comme catégorie de l'expérience permettant d'intégrer, de structurer, d'interpréter les situations et les événements du vécu, l'article se donne pour objet d'examiner la notion d'expérience biographique dans son rapport aux situations et aux objets de formation. La manière dont les individus biographient leurs expériences, et au premier chef la manière dont ils intègrent dans leurs constructions biographiques ce qu'ils font et ce qu'ils sont dans leur famille, à l'école, dans leur profession, en formation continue, est partie prenante du processus d'apprentissage et de formation. Dans l'activité de biographisation se jouent la rencontre, la négociation, la perlaboration entre les projets de soi dont sont porteurs les individus et les projets collectifs véhiculés par les instances de formation.

Based on an analysis of biography as a category of experience which enables a person to integrate, structure and interpret past situations and events, this paper examines the notion of the "biographical experience" in relation to situations and educational objectives. The way in which individuals "biographize" (i.e. turn into a narration) their experiences - and more importantly the way in which they integrate into their biographical constructions what they do, their role within the family, at school, at work, on adult training courses - plays a large part in the learning and training process. The conjunction, negotiation and "working-through" of individuals self - projects and of collective projects of educational or training institutions play a central part in this "biographization" process.

\section{INDEX}

Keywords : Biographic Experience, Biographization, Biography, Development, Socialization Mots-clés : Biographie, Biographisation, Expérience biographique, Formation, Socialisation

\section{AUTEUR}

\section{CHRISTINE DELORY-MOMBERGER}

Maître de conférences en sciences de l'éducation à l'Université Paris 13/Nord (Département des sciences de l'éducation, 99, avenue Jean-Baptiste Clément, 93430 Villetaneuse). Courriel :

delbourg@club-internet.fr 